\title{
Publications received
}

Hidrogeotecnia nas Barragens : metodo de analise, par Roberto Monteiro de Andrade. March 1984, published by Engevix S.A., in Portuguese.

In continuation to previous works the author develops a method for analysis of uplift forces in the foundations of hydraulic structures. The method is based on equilibrium of nux on each node of a flow mesh and for that reason is called MEPON-Metodo dos Pontos Nodais (Nodal Point Method)

More than 45 examples help the reader to understand from elementary to sophisticated tridimensional hydrogeotechnical phenomena.

The method is well suited for personel computers and two programs in BASIC ATARI are presented.

The method allowed a critical analysis of the drainage systems usually adopted to reduce uplift forces. It is shown that drainage curtains drilled deeper than necessary may lead to an increase in uplift

Piezometric data from Palmar (Lruguai), Balsas Mineiro (Brazil) and Itaipu (Brazil-Paraguai) Hydropower Plants are analysed and interpreted by means of the method.

Buried rigid pipes, structural design of pipelines, by O.C. Young and J.J. Trott. 234 pp.. Elsevier Publishers.

Les granulats ma,ins, par $C$. Augris et P. Cressard. Publications Ifremer, 1984. 90 pp. en Français. En vente à : BNDO Documentation, B.P. 337, 29273 Brest Cedex, France, 70 francs français.

Lurbanisation, la protection des sites et la sauvegarde de nappes deau souterraines réduisent les zones dexploitation de granulats alluvionnaires. La mer offre des possibilites de substitution. Les granulats marins constituent en France un potentiel appreciable dans l'approvisionnement en matériaux des régions côtières. De nombreuses campagnes a la mer sur le plateau continental français mettant en œuvre différents moyens de prospection (sismique, carottage, dragage) et techniques d'identification ont permis au CNEXO de dresser linventaire des ressources et de mettre en évidence des gisements exploitables. L'exploitation des granulats en France est répartie en Manche et en Atlantique. Elle est tributaire de divers facteurs: éléments techniques, impact sur l'environnement et donnèes économiques. Le dragage des granulats marins est soumis à la législation minière. Les entreprises qui exploitent ce type de matériaux représentent 29 armements comptant 40 navires sabliers. D'autres pays utilisent les matériaux marins pour leurs besoins; parmi eux, la Grande-Bretagne est l'un des plus importants producteurs.

\section{Sommaire}

Le potentiel français

I. Recherche et valorisation

1. Origine et mise en place

2. Méthodes de prospection.....

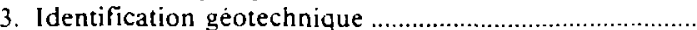

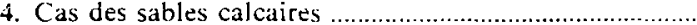

5. Ressources et réserves : notion évolutive.

II. Inventaire par région

L'exploitation des granulats marins

I. Techniques d'exploitation

1. Les dragues.

2. Transport et déchargement des matériaux

3. Contraintes du dragage

II. Dragage et environnement

1. Analyse du phénomène

2. Une expérience originale :

la souille expérimentale de la baie de Seine....

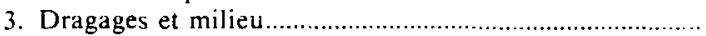

1II. Aspects économiques

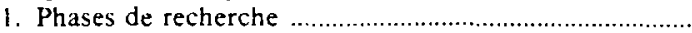

2. La trilogie : extraction - transport - déchargement .........
La situation dans divers pays

I. En France

1. La legislation

2. Les activites

3. L’armement sablier français

II. La Grande-Bretagne

III. Autres pays

Suggested methods for surface monitoring of movements across discontinuities, ISRM, Commission on testing methods. Orders from ISRM Secretariat, LNEC, 101, ave do Brasil, P-1799, Lisboa Codex, Portugal. 3 U.S. S for non-members.

Method 1: monitoring movement across discontinuities using glass plates. Method 2: monitoring movement across discontinuities using pins and a tape. Method 3: monitoring movement across discontinuities using portable mechanical gauge. Method 4: monitoring movement across discontinuities using a remote reading electrical jointmeter.

Geologia applicata e idrogeologia. Proceedings of the 8 th Salt Water Intrusion Meeting (Bari, 25-29 May 1983) : published by the "Istituto di Geologia applicata e geotecnica dell" Universita di Bari”. Italy. 91 contributions, $394 \mathrm{pp}$. in English.

Problems of geomechanics, 1984, no 9, 159 pp., published by the Academy of Sciences of the Armenian S.S.R.. Institute of Geophysics and Engineering seismology ; 13 papers, in Russian with English and Armenian abstracts.

Beginning of the Quinary or Technogene, an engineering geological analysis, by G. Ter-Stepanian, published by the Armenian Academy of Sciences, Yerevan. Armenia, URSS : 100 pages, in Russian with an English abridgement (10 pages).

Catastrofes naturales, a publication of the "Catedra de geologia aplicada a las obras publicas", Universidad Politecnica de Valencia, May 1985, 75 pp., in Spanish.

AGID News, official Newsletter of the Association of geoscientists for international development. No 44, July 1985.

News of the International Society for Rock Mechanics. No 75 , 1985, July-September.

ISSMFE News, vol. 12, n², June 1985.

Jornal da ABGE (Associação Brasileira de Geologia de Engenharia), July-August 1985, no 36.

Southeast Asian Geotechnical Society, Circular no $85 / 2$.

New Zealand Geomechanics News, no 30 , July 1985.

Journal of the Japan Society of Engineering Geology, vol. 26, no $2,1985$.

"Episodes", International Geoscience Newsmagazine, published by the International Union of Geological Sciences, vol. 8, no 3, September 1985. 